\title{
Malignant priapism secondary to isolated penile metastasis from a renal pelvic carcinoma
}

\author{
Sulai Liu, MD, ${ }^{*}$ Fuhua Zeng, MD; ${ }^{\dagger}$ Lin Qi, MD, PhD; ${ }^{*}$ Shusuan Jiang, MD, ${ }^{\dagger \xi}$ Pingping Tan, MD; ${ }^{*}$ Xiongbing Zu, \\ $M D ;{ }^{*} Y u$ Xie, MD; Weiqing Han, $M D^{\dagger}$
}

*Department of Urology, Xiangya Hospital, The Central South University, Changsha China; 'Department of Urology, The Affiliated Tumor Hospital of Xiangya Medical School, The Central South University, Changsha, China; §Department of Urology, The first affiliated hospital of Guangxi Medical University, Nanning, China; *Department of Pathology, The Affiliated Tumor Hospital of Xiangya Medical School, The Central South University, Changsha, China

Cite as: Can Urol Assoc J 2014;8(5-6):e558-60. http://dx.doi.org/10.5489/cuaj.1695 Published online August 11, 2014.

\section{Abstract}

We report a case of isolated penile metastasis from renal pelvic carcinoma in a 69-year-old man with malignant priapism. The patient had radical resection of the renal pelvic carcinoma 2 months earlier with urothelial carcinoma (UC) and sarcomatoid differentiation histology. Physical examination showed no visible skin lesions, but a palpable hard nodule was present over the penile shaft. The imaging studies did not reveal other metastases. Cavernous-Glans shunt and nodule resection were performed, and histological examination showed metastasis UC with sarcomatoid differentiation. To our knowledge, we describe the first case of malignant priapism due to isolated penile metastasis of renal pelvic carcinoma.

M alignant priapism is prolonged penile erection without sexual stimuli, and is generally related to medical treatment, sickle cell disease, leukemia, polycythemia, penile metastasis of malignancies, pelvic thrombophlebitis and neurological diseases. ${ }^{1-3}$ Metastasis to the penis mimicking priapism is extremely rare, particularly in the absence of disseminated disease. About 300 cases have been reported. ${ }^{4}$

Primary sites include the bladder (33\%), prostate $(30 \%)$, colon (17\%) and kidney (7\%). Extra-pelvic sites, including the lung, pancreas, stomach, esophagus, melanoma and testis, have been noted..$^{6-9}$ Fewer than 20 cases of penile metastasis from renal cancer have been reported, most of them were renal cell carcinoma in origin. ${ }^{10,11}$ On the other hand, penile metastasis has been a part of more widespread disease in about $90 \%$ of reported cases. ${ }^{12}$ We describe a case of malignant priapism caused by penile metastasis of renal pelvic carcinoma in the absence of radiological disseminated disease. There have been no previous reports of renal pelvic carcinoma without regional metastasis with only penis metastasis. This is the first case of a renal pelvic carcinoma with isolated metastasis to penis.

\section{Case report}

A 69-year-old male presented to our clinic with a 2-week history of malignant priapism. He had a radical resection of the renal pelvic carcinoma for right renal pelvic carcinoma at an outside centre 2 months previously (Fig. 1) and the histopathology examination of the resected specimen revealed urothelial carcinoma (UC) and sarcomatoid differentiation (Fig. 2). All surgical margins and circum-renal lymph nodes removed during surgery were negative for tumour. The patient did not receive any kind of postoperative adjuvant therapy and was in a good general condition. On physical examination, the penis was tender, erect and consistency was wood-like, but the glans was flaccid. There was a palpable nodule in the corpora cavernosa of the penis, but no overlying skin necrosis of the glans. Corporal fine needle aspiration for blood-gas analysis failed to obtain any confirmatory sample. On digital rectal examination there was no suspicion of tumour involvement. Results of the laboratory evaluations were: white blood cell $9.52 \times 109 / \mathrm{L}$; red blood cell $3.78 \times 1012 / \mathrm{L}$; platelet $278 \times 109 / \mathrm{L}$; hemoglobin $140 \mathrm{~g} / \mathrm{L}$; prostate-specific antigen $2.74 \mathrm{ng} / \mathrm{mL}$. There were also no abnormal findings on urinalysis. There were no other abnormal changes on the intravenous urogram (IVU) and cystourethroscopy. Full radiological evaluation with computed tomography failed to reveal any other evidence of disease in thorax, abdomen and pelvis. His bone scan was negative. Cavernous-Glans shunt (Winter) was performed, and at the same time, an incisional biopsy of the penile nodule at the corpus cavernosum was taken for tissue diagnosis, which later confirmed metastasis UC with sarcomatoid differentiation (Fig. 3).

Three days later after the procedure, the patient's symptoms improved. He and his family decided against surgical amputation. 


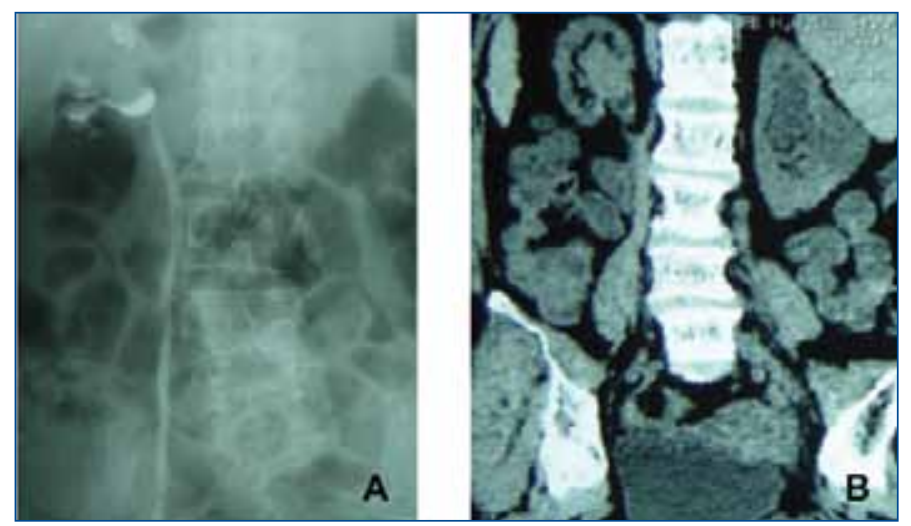

Fig. 1. Imaging examination showed right renal pelvic carcinoma. A: Retrograde pyelography, the right renal pelvis filling defect. B: A computed tomography of the right renal pelvis showing the solid portions density.

\section{Discussion}

Sarcomatoid carcinoma is considered a high-grade UC. The prognosis for sarcomatoid carcinoma of the renal pelvis is poor, as it is usually high grade at diagnosis. Several cases of sarcomatoid UC have been reported..$^{13,14}$

Immunohistochemical studies of the spindle cell component have demonstrated frequent expression of cytokeratin (CK), at least focally, as well as the expression of other epithelial markers, such as epithelial membrane antigen (EMA), but less consistently than CK. ${ }^{15}$ Coexpression of vimentin is common, with muscle-specific actin infrequently expressed. ${ }^{13,14}$ Immunohistochemical studies demonstrating biphasic differentiation (e.g., examinations of cytokeratin and vimentin expression revealed epithelial and mesenchymal differentiation, respectively) were also mandatory in the current diagnosis.

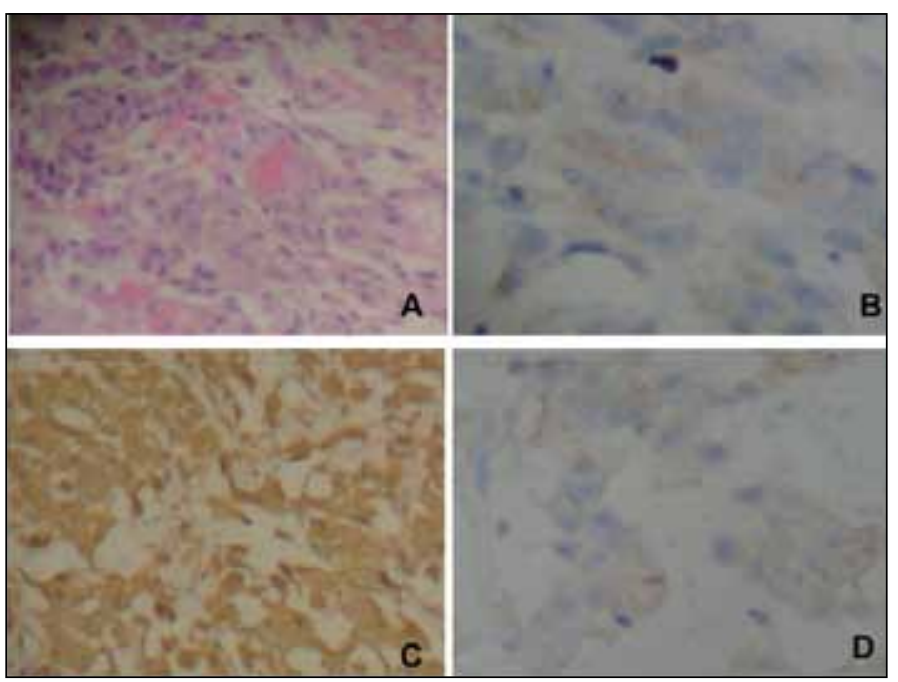

Fig. 3. The histopathologic examination of the penile nodule revealed metastatic renal pelvic carcinoma with sarcomatoid differentiation. A: Hematoxylin and eosin (H\&E) staining $\times 200$ immunohistochemistry; B: vimentin $($ Vim $) \times 200$; : cytokeratin pan $(C K) \times 200$; D: epithelial membrane antigen $(E M A) \times 200$.
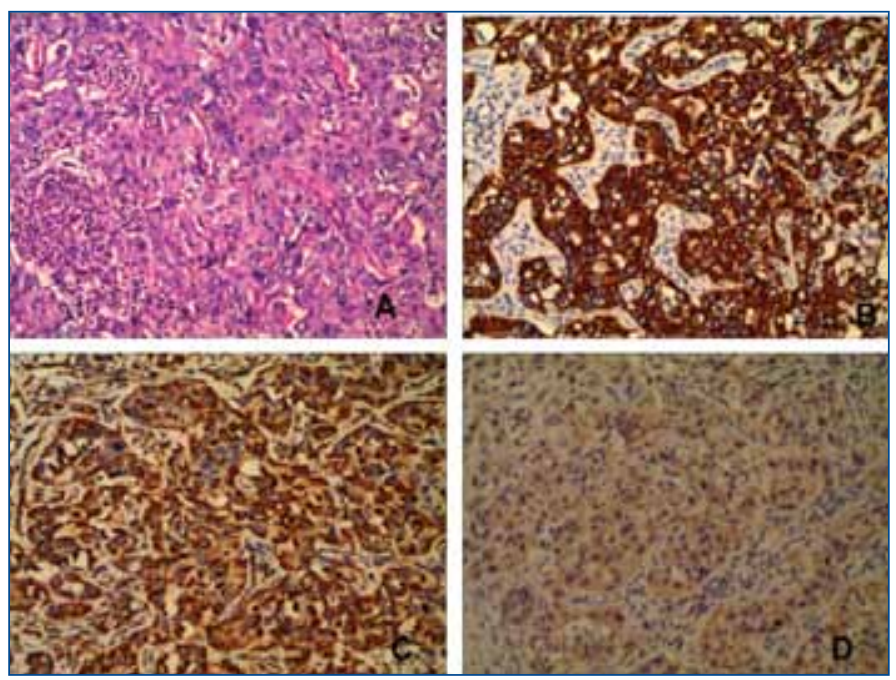

Fig. 2. The histopathologic examination of therenal pelvic carcinoma with sarcomatoid differentiation. A: Hematoxylin and eosin (H\&E) stain $\times 100$ immunohistochemistry; B: cytokeratin $(C K) \times 100$; C: vimentin $(V i m) \times 100$; D: epithelial membrane antigen, $(E M A) \times 100$.

A sarcomatoid renal pelvic tumour of UC origin should not be confused with sarcomatoid renal cell carcinoma, which is a well-described, high-grade malignant variant of renal parenchymal origin. In contrast, a sarcomatoid renal pelvic tumour of UC origin is composed of epithelial and spindle cell populations. Figure 2 shows a malignant spindle cell neoplasm containing non-neoplastic multinucleated osteoclast-type giant cells with hematoxylin-eosin staining and strongly positive cytokeratin immunostaining. This figure highlights the neoplastic spindle cells, confirming their epithelial nature.

Penile metastasis from other primary sites is rare. About $40 \%$ of them are manifested with malignant priapism, a painful penile erection secondary to tumour metastasis. ${ }^{1}$ Priapism occurs due to tumour infiltration of the corpora cavernosa and/or the venous drainage. ${ }^{3}$ There are about 300 published cases of secondary penile malignancies. Most of the primary lesions are prostate and bladder, with the recto sigmoid region contributing to the bulk of the remaining cases. ${ }^{2}$ To our knowledge, this is the first case of penile metastases from renal pelvic carcinoma.

It is not clear why penile metastasis does is infrequent, despite the fact that the penis has rich vascularazition and intensive venous communication with neighbouring organs. Various mechanisms for penile metastasis have been suggested, which include retrograde venous spread, retrograde lymphatic spread, arterial spread, direct extension, implantation and secondary to instrumentation. ${ }^{9}$ Accumulating clinical evidence indicates that retrograde venous spread from the pudendal into the dorsal venous system of the penis is the most likely mechanism. About two-thirds of all penile metastasis are detected at a mean time of 18 months after 
Liu et al.

the detection of the primary tumour; the remaining third present at the same time as the primary tumour. ${ }^{16}$ In our case, penile metastasis developed 2 months after radical resection of the renal pelvic carcinoma, unusually as the first manifestation of malignant priapism. The penile metastasis may have developed at the same time as the primary renal pelvic carcinoma, but the penile region was not considered.

Penile metastases can represent a late development in the clinical course of metastatic carcinoma; most patients had metastatic disease elsewhere before metastasis developed in the penis. ${ }^{1-3,9,16}$ More than $80 \%$ of patients die within 6 months, irrespective of the primary tumour and treatment. ${ }^{16}$ However, in our case, the imaging studies did not show other metastases; therefore, we concluded that the penis was the isolated metastatic site. This was extremely rare. ${ }^{17}$

\section{Conclusion}

Penile metastasis may rarely be the primary presentation of an occult cancer. We report the first case of malignant priapism secondary to isolated penile metastasis of renal pelvic carcinoma, which illustrates the clinical feature of isolated penile metastasis of renal pelvic carcinoma. Clinicians should be aware of this rare entity and adopt appropriate strategies of treatment that can achieve the best outcome for patients.

Acknowledgements: This work was supported by Hunan Provincial Innovation Foundation for Postgraduate (NO. 250171380100015) and supported by the Fundamental Research Funds for the Central Universities of Central South University (NO. 217772150050582).

Competing interests: Dr. Liu, Dr. Zeng, Dr. Qi, Dr. Jiang, Dr. Tan, Dr. Zu, Dr. Xie and Dr. Han all declare no competing financial or personal interests.

This paper has been peer-reviewed.

\section{References}

1. Witters S, Cornelissen M, Vereecken RL. Malignant priapism. Eur Urol 1985;11:431-2.

2. Lin YH, Kim JJ, Stein NB, et al. Malignant priapism secondary to metastatic prostate cancer: A case report and review of literature. Rev Urol 2011;13:90-4.

3. Hamamoto S, Tozawa K, Nishio $\mathrm{H}$, et al. Successful treatment of primary malignant lymphoma of the penis by organ-preserving rituximab-containing chemotherapy. Int I Clin Oncol 2012;17:181-4. http:// dx.doi.org/10.1007/s10147-011-0273-8

4. Hizli F, Berkmen F. Penile metastasis from other malignancies. A study of ten cases and review of the literature. Urol Int 2006;76:118-21. http://dx.doi.org/10.1159/000090872

5. Barocas DA, Chang SS. Penile cancer: Clinical presentation, diagnosis, and staging. Urol Clin North Am 2010;37:343-52. http://dx.doi.org/10.1016/j.ucl.2010.04.002

6. Pai A, Sonawane S, Purandare NC, et al. Penile metastasis from esophageal squamous carcinoma after curative resection. Ann Thorac Cardiovasc Surg 2008; 14:238-41.

7. Kurul S, Aykan F, Tas F. Penile metastasis of cutaneous malignant melanoma: A true hematogenous spread? Case report and review of the literature. Melanoma Res 2006;16:259-61. http://dx.doi. org/10.1097/01.cmr.0000215032.15565.cc

8. Ahn TY, Choi EH, Kim KS. Secondary penile carcinoma originated from pancreas. J Korean Med Sci 1997; 12:67-9.

9. Cherian J, Rajan S, Thwaini A, et al. Secondary penile tumours revisited. Int Semin Surg Oncol 2006;3:33. http://dx.doi.org/10.1186/1477-7800-3-33

10. Pascual Mateo $C$, Nieto Gallo MA, Lujan Galan $M$, et al. Acute urinary retention and hematuria secondary to metastatic renal clear-cell carcinoma to the penis. Actas Urol Esp 2005;29:593-5. http://dx.doi. org/10.1016/S0210-4806(05)73302-7

11. Daniels GF Jr., Schaeffer AJ. Renal cell carcinoma involving penis and testis: Unusual initial presentations of metastatic disease. Urology 1991;37:369-73. http://dx.doi.org/10.1016/0090-4295(91)80269-D

12. Dubocq FM, Tefilli MV, Grignon DJ, et al. High flow malignant priapism with isolated metastasis to the corpora cavernosa. Urology 1998;51:324-6. http://dx.doi.org/10.1016/S0090-4295(97)00607-9

13. Thiel DD, Igel TC, Wu KJ. Sarcomatoid carcinoma of transitional cell origin confined to renal pelvis. Urology 2006;67:622 e9-11.

14. Canacci AM, MacLennan GT. Sarcomatoid urothelial carcinoma of the renal pelvis. J Urol 2006;175:1906. http://dx.doi.org/10.1016/S0022-5347(06)00431-9

15. Vermeulen P, Hoekx L, Colpaert C, et al. Biphasic sarcomatoid carcinoma (carcinosarcoma) of the renal pelvis with heterologous chondrogenic differentiation. Virchows Arch 2000;437:194-7. http://dx.doi. org/10.1007/s004280000190

16. Pomara $G$, Pastina I, Simone $M$, et al. Penile metastasis from primary transitional cell carcinoma of the renal pelvis: First manifestation of systemic spread. BMC Cancer 2004;4:90. http://dx.doi. org/10.1186/1471-2407-4-90

17. Arya M, Kalsi J, Kelly J, et al. Malignant and premalignant lesions of the penis. BMJ 2013;346:f1 149. http://dx.doi.org/10.1136/bmi.f1149

Correspondence: Dr. Fu-Hua Zeng, Department of Urology, The Affiliated Tumor Hospital of Xiangya Medical School, The Central South University, 283 Tongzipo Road, Changsha, Hunan 410013, China; zfh_urologist@163.com; and Dr. Lin Qi, The Department of Urology, Xiangya Hospital, Central South University, 87 Xiangya Road, Changsha, Hunan 410008, China; urinologist_xyq@163.com 